\title{
Age-related prevalence of non-cardiac surgery indications
}

\author{
Nera Agabiti \\ Department of Epidemiology, Regional Health Service Lazio, Rome, Italy
}

\begin{abstract}
A clear trend in Italy over the last 20 years has been the increasing prevalence of "very old" people (over 75 years of age) within the general population. Routine statistics as well as population surveys and ad hoc epidemiological studies all confirm this trend. The proportion of socalled "very old patients in good health" is the category which is increasing the most. This is due to the economic well-being and generally good living conditions in Italy as well as progress in medical care and the introduction of new treatments and technologies in clinical practice that have improved survival and quality of life. The presence of an increasing number of very old people in the population, with consequent change of the population's health needs, represents a great challenge both for the healthcare system and for society as a whole.
\end{abstract}

\section{Importance of the issue and epidemiological data}

Based on ISTAT (Italian National Bureau of Statistics), Italy has $6,828,610$ individuals who are aged over 75 years, corresponding to $11 \%$ of the total population, and more than 4.7 million elderlies aged between 75 and 84 years, representing as much as $7.8 \%$ of the total population. The group of the "oldest old" (aged $\geq 85$ years) comprises more than 1.9 million individuals, corresponding to $3.2 \%$ of the total population living in Italy. The category of the "oldest old" is the one showing the greatest increase over the years, passing from $3.5 \%$ (of the $>65$ years of age) in 2010 to $5.2 \%$ in 2016 [1-3]. Among the conditions of surgical risk in the elderly, one of the most frequent is hip fracture. In Italy, in 2014, there were reported 117,436 hospital admissions for hip fracture, $80 \%$ of which were among people aged $>75$

Corresponding author: Nera Agabiti, Department of Epidemiology, Regional Health Service Lazio, Via Cristoforo Colombo 112, Roma, Italy. E-mail: n.agabiti@deplazio.it

Key words: Age; non-cardiac surgery; prevalence; public health; risk factors.

Received for publication: 25 November 2016

Accepted for publication: 5 December 2016

CC Copyright N. Agabiti, 2017

Tipografia PI-ME Editrice, Italy

Monaldi Archives for Chest Disease 2017; 87:839

doi: 10.4081/monaldi.2017.839

This article is distributed under the terms of the Creative Commons Attribution Noncommercial License (by-nc 4.0) which permits any noncommercial use, distribution, and reproduction in any medium, provided the original author(s) and source are credited. years [4]. Data on the clinical characteristics of patients who undergo non cardiac surgery are available in the international literature. Guidelines recommend standardized management protocols for elderly surgery patients and diverse strategies have been proposed for assessing the preoperative risk in the more advanced ages [5-8]. Also in Italy, several studies have documented the impact of elderly age in non cardiac surgery and, in particular, the problem of comorbidity [2,9]. A recent epidemiological study reported the clinical characteristics of patients admitted for hip fracture between 2007 and 2010 to hospitals in the Lazio region, based on data from the Hospital Information System (Sistema Informativo Ospedaliero, SIO). The prevalence of diabetes was $6.1 \%$, heart disease $21.2 \%$, cerebrovascular disease $14.7 \%$, hypertension $30.7 \%$, diseases of the central nervous system $11.6 \%$, chronic kidney disease (CKD) 6.3\%, and chronic obstructive pulmonary disease (COPD) 6.3\%. These comorbidities played a significant role both in reducing the probability of early surgery (considered fundamental for a good prognosis, but overall only $20.3 \%$ were operated within 48 hours) - as well as an adverse prognostic factor for mortality at 30 days (overall 30-day mortality 7.5\%) [10]. In a larger sample of patients with hip fracture identified by the regional Hospital Information System (SIO Lazio) in the years 2007-2012, a difference between patients operated $v s$ not operated was found regarding the concomitant presence of comorbidity, e.g. heart failure was present in $9 \%$ of the patients not operated for hip fracture $v s 5 \%$ of those operated. The same pattern was found for cardiac arrhythmias (10.6\% vs 7.1\%), CKD (9.1\% vs 4.8\%), COPD (8.4\% vs 4.7\%), dementia (12.5\% vs $8.3 \%)$, hypertension ( $17.3 \%$ vs $3.0 \%)$, diabetes (9.3\% vs $5.9 \%)$, and ischemic heart disease (13.5\% vs $7.9 \%)$ [11].

The role of the clinical complexity of elderly patients in non cardiac surgery was also analyzed in a cohort of patients who underwent cholecystectomy in the period 2007-2008. The proportion of comorbidities increased by $20 \%$ in the population aged $\geq 75$ years compared to the total population studied. Also for this type of surgery, the factor of age combined with comorbidity resulted in an increased risk of adverse events post-surgery. Cardiovascular and systemic complications were $7.1 \%$ in the $80+$ age-group versus $1.5 \%$ in the under-70 agegroup. Regarding surgery-related complications, the differences were less pronounced (3.3\% among the $80+$ age-group versus $1.8 \%$ in the under-70 age-group). The study also showed that the superiority of laparoscopy over laparotomy in terms of post-surgical complications - evident in the total population sample - did not differ significantly between the youngest and the very old [12]. This finding is in line with other reports [13]. A recent systematic review on the efficacy of the ERAS protocol in bowel surgery suggests the efficacy of the same protocol of perioperative management in terms of outcomes (morbidity, mortality, and length of stay in hospital post-surgery) without substantial differences between the youngest and very old [14]. Analysis of pharmacological data for the region of Lazio, which gathers data related to pharmacological prescriptions delivered by public and private pharmacies of the Region, showed of prevalence of pharmaceutical drug use in the 65+ age-group in 2010 [15]. Of the 1,122,864 individuals examined, $57.9 \%$ were women and $48.8 \%$ were in the $75+$ age- 
group. Approximately $30 \%$ had been admitted to hospital in the two years prior; $6.8 \%$ resulted as not having received any pharmacological prescription in the period surveyed, while $58.8 \%$ were taking 6 or more drugs. Among those using 6 or more classes of drugs in the period, approximately $60 \%$ were women and more than half were aged over 75 years. Among the drugs used, proton pump inhibitors were the most frequent (persons with at least one prescription 46.6\%), followed by statins (28.5\%), antiplatelet drugs (34.7\%), ACE inhibitors (22\%), calcium channel blockers (20.6\%), and angiotensin II receptor antagonists (18.4\%).

\section{Considerations}

\section{For the policy-maker}

The changing characteristics of society over the course of time require that policy-makers be attentive to these changes and meet the new health needs. The increase of elderly who are "in good health" and live alone calls for the development of a more efficient social network that can support them, facilitate the management of daily life activities and enable their active participation in social life. On the other hand, the presence of "many elderlies affected by invalidating diseases" represents a burden for their families, who ask for greater support from society. From the point of view of health planning, already for a number of years a special effort is being made at the level of national and regional legislation to put the problematics of the "very old" patient at the center of the new health organization models, facilitating access to appropriate and effective care paths also for this age-group. The quality assessment of health services, a key aspect of health politics, has recently given impulse to the development of screening methods and tools which take into account the "very old" age-group and the need to adapt the offer of services to the changing needs of the population, in respect of the principle of universal and equal protection of health of our National Health System.

\section{For the physician}

Physicians are being confronted more and more frequently in their daily practice with complex clinical cases, i.e. patients in very old age who present as candidates for sophisticated medical treatments (e.g. surgical interventions or invasive procedures) with a good probability of a favorable outcome. In the case of surgery, the specialist surgeon must be able to draw on a multidisciplinary network in order to manage the postoperative course with special attention, identifying the risks of organ failure (respiratory, cardiac, or renal) to which the patients are exposed on account of their global clinical picture. Moreover, the intraoperative management of very old patients with multi-comorbidities often poses problems of a medical and ethical nature. The improvement both of patients' "baseline" health conditions as well as in the surgical techniques in the different settings - with approaches aimed at reducing the surgical invasiveness - has led physicians to expand the concept of operability, in particular in fields where tissue removal (e.g. lung cancer) or the control of dysfunction (e.g. heart valve disease with an interventionist hemodynamic approach) can reduce symptoms and increase the survival. A significant challenge, finally, is the communitybased assistance (local districts, GPs) for complex patients (e.g. with organ failure) which should be complementary - in a vision of integrated hospital-community care - to the intensive medical care provided in hospital. The continuative and efficient management of the patient should always take into account the needs of the patient and their family in a perspective of a shared participation (between physician and patient) in the disease and in its treatment.

\section{For the patient/citizen}

The availability of information about health and health treatments at relatively low cost (through internet, newspapers, publicity) has changed considerably in the last few years the awareness of health problems on the part of citizens/patients. This fact has had repercussions on the physician-patient relation, which has in fact evolved with respect to past decades, when the doctor was considered by the patient as the sole possessor of "truth" about the disease and its treatment. Today one speaks much about "values" in healthcare, and among them the first and foremost is the respect for the patient's needs and wishes. The very old patient with multi-comorbidities has to often take numerous different therapies. It is well known that the more pharmaceutical drugs there are prescribed, the less adherent the very old patient tends to be. Difficult also is the management of devices which are proposed on the market as important instruments of care (e.g. inhaled sprays in COPD). For their correct use, a strong motivation and understanding of their efficacy are necessary on the part of the patient and family. On the doctor's side, great attention to these aspects and time must be dedicated. It should also be highlighted that non pharmacological treatments (e.g. nutrition, adequate mobilization) play a fundamental role in the elderly patient.

\section{For scientific research}

The superiority of randomized controlled trials (RCTs) in assessing the comparative efficacy of treatments has been undermined, in recent times, in the context of a recent methodological scientific discussion. The design of observational studies, often based on data of the current information systems, is gaining more and more ground within the scientific community, creating the opportunity to integrate the results of the RCTs with data coming directly from clinical practice. Observational studies have the potential, moreover, to investigate an important aspect, i.e. the efficacy of pharmacological and non pharmacological interventions, in categories of population which by definition are not considered by RCTs, e.g. the elderly and the very old. Hence, considerable contributions are expected from these new design approaches and analyses to estimate the outcomes of healthcare interventions in the elderly population and the very old and respond to scientific questions that are still open in many sectors of diagnosis and treatments.

\section{References}

1. ISTAT. Bilancio demografico nazionale. http:/www.istat.it/it/archivio/ demografia

2. Dipartimento di Epidemiologia SSR Lazio. Stato di salute della population a Roma e in Lazio. Available from: http:/www.deplazio.net/it/ stato-di-salute

3. Osservatorio Nazionale sulla Salute nelle Regioni Italiane. Available from: http://www.osservasalute.it/

4. Programma Nazionale Esiti (PNE). Available from: http://95.110.213. 190/PNEed15/index.php

5. Griffiths R, Beech F, Brown A, et al. Peri-operative care of the elderly 2014: Association of Anaesthetists of Great Britain and Ireland. Anaesthesia 2014;69(Suppl 1):81-98.

6. Fleisher LA, Fleischmann KE, Auerbach AD, et al. 2014 ACC/AHA guideline on perioperative cardiovascular evaluation and management of patients undergoing noncardiac surgery: executive summary: a report of the American College of Cardiology/American Heart Association Task Force on Practice Guidelines. Circulation 2014;130):2215-45. 
7. Velasco A, Reyes E, Hage FG. Guidelines in review: Comparison of the 2014 ACC/AHA guidelines on perioperative cardiovascular evaluation and management of patients undergoing noncardiac surgery and the 2014 ESC/ESA guidelines on noncardiac surgery: Cardiovascular assessment and management. J Nucl Cardiol 2016 Aug 22.

8. Devereaux PJ, Sessler DI. Cardiac complications in patients undergoing major noncardiac surgery. N Engl J Med 2015;373):2258-69.

9. Programma Regionale di Valutation degli Esiti (P.Re.Val.E.). Available from: http://95.110.213.190/prevale2016/index.php

10. Colais P, Agabiti N, Fusco D, et al. Inequality in 30-day mortality and the wait for surgery after hip fracture: the impact of the regional health care evaluation program in Lazio (Italy). Int J Qual Health Care 2013;25:239-47.
11. Colais P, Di Martino M, Fusco D, et al. The effect of early surgery after hip fracture on 1-year mortality. BMC Geriatr 2015;15:141.

12. Agabiti N, Stafoggia M, Davoli M, et al. Thirty-day complications after laparoscopic or open cholecystectomy: a population-based cohort study in Italy. BMJ Open 2013;3 pii: e001943.

13. Antoniou SA, Antoniou GA, Koch 00, et al. Laparoscopic colorectal surgery confers lower mortality in the elderly: a systematic review and meta-analysis of 66,483 patients. Surg Endosc 2015 Feb;29:322-33.

14. Bagnall NM, Malietzis G, Kennedy RH, et al. A systematic review of enhanced recovery care after colorectal surgery in elderly patients. Colorectal Dis 2014;16:947-56.

15. Kirchmayer U, Mayer F, Basso M, et al. Polypharmacy in the elderly: a population based cross-sectional study in Lazio, Italy. Eur Geriatr Med 2016. Available from: http://dx.doi.org/1'.1016/j.eurger.2016.05.008 